\title{
Some identities and reciprocity relationsof unipoly-Dedekind type DC sums
}

Hye Kyung Kim" ${ }^{*}$ and Dae Sik Lee ${ }^{2}$

"Correspondence: hkkim@cu.ac.kr
'Department of Mathematics
Education, Daegu Catholic
University, Gyeongsan 38430,
Republic of Korea
Full list of author information is
available at the end of the article

available at the end of the article

\begin{abstract}
Dedekind type DC sums and their generalizations are defined in terms of Euler functions and their generalization. Recently, Ma et al. (Adv. Differ. Equ. 2021:30 2021) introduced the poly-Dedekind type DC sums by replacing the Euler function appearing in Dedekind sums, and they were shown to satisfy a reciprocity relation. In this paper, we consider two kinds of new generalizations of the poly-Dedekind type DC sums. One is a unipoly-Dedekind type DC sum associated with the type 2 unipoly-Euler functions expressed in the type 2 unipoly-Euler polynomials using the modified polyexponential function, and we study some identities and the reciprocity relation for these unipoly-Dedekind type DC sums. The other is a unipoly-Dedekind sums type DC associated with the poly-Euler functions expressed in the unipoly-Euler polynomials using the polylogarithm function, and we derive some identities and the reciprocity relation for those unipoly-Dedekind type DC sums.
\end{abstract}

MSC: 11F20; 11B68; $11 \mathrm{~B} 83$

Keywords: Dedekind type DC sums; Type 2 poly-Euler polynomials; Type 2 poly-Genocchi polynomials; Poly-Euler polynomials; Euler function; Polylogarithm function

\section{Introduction}

Let

$$
((x))=\left\{\begin{array}{ll}
x-[x]-\frac{1}{2}, & \text { if } x \notin \mathbb{Z}, \\
0, & \text { if } x \in \mathbb{Z},
\end{array} \quad \text { (see }[1-8,20-28,30]\right),
$$

where [·] denotes the greatest integer not exceeding $x$.

The Dedekind sums are defined by

$$
S(h, m)=\sum_{\mu=1}^{m-1}\left(\left(\frac{\mu}{m}\right)\right)\left(\left(\frac{h \mu}{m}\right)\right) \quad(\text { see }[1-8,20-28,30]),
$$

where $h$ and $m$ are positive integers.

C) The Author(s) 2021. This article is licensed under a Creative Commons Attribution 4.0 International License, which permits use, sharing, adaptation, distribution and reproduction in any medium or format, as long as you give appropriate credit to the original author(s) and the source, provide a link to the Creative Commons licence, and indicate if changes were made. The images or other third party material in this article are included in the article's Creative Commons licence, unless indicated otherwise in a credit line to the material. If material is not included in the article's Creative Commons licence and your intended use is not permitted by statutory regulation or exceeds the permitted use, you will need to obtain permission directly from the copyright holder. To view a copy of this licence, visit http://creativecommons.org/licenses/by/4.0/. 
The most fundamental result in the theory of Dedekind sums is the reciprocity theorem. If $h$ and $m$ are relatively prime positive integers, then

$$
S(h, m)+S(m, h)=-\frac{1}{4}+\frac{1}{12}\left(\frac{h}{m}+\frac{m}{h}+\frac{1}{h m}\right), \quad(\text { see }[2]) .
$$

It is well known that the classical Dedekind sums $S(h, m)$, initiated by Richard Dedekind [9], first arose in the transformation formula of the logarithm of the Dedekind eta function. Dedekind introduced them to express the functional equation of the Dedekind eta function. These sums have figured prominently in so many different areas such as elliptic modular functions to number theory, analysis, number theory, combinatorics, q-series, Weierstrass elliptic functions, modular forms, and other areas $[1-9,13,14,16,18,20-$ 30]. In combinatorial number theory, one is interested in partitions of an integer $n$ from a finite set of positive integers. Beck et al. showed that the number of such partitions of $n$ from a finite set is a quasi-polynomial in $n$, whose coefficients are built up from some generalization of Dedekind sums [3]. Bayad and Simsek [4,28] studied three new shifted sums of Apostol-Dedekind-Rademacher type. The Dedekind type DC (Daehee and Changhee) sums using Euler functions were first introduced by Kim [13] and have been studied variously by several authors since then [20,30]. Recently, as a generalization of Dedekind sums, the poly-Dedekind sums associated with the type 2 poly-Bernoulli functions of in$\operatorname{dex} k$ [18] and the unipoly-Dedkind sum [11] were introduced. In addition , Ma et al. introduced the poly-Dedekind sums associated with the poly-Bernoulli functions of index $k$ [21] and the poly-Dedekind type DC sums associated with the type 2 poly-Euler functions of index $k$ [20].

In this paper, we introduce two kinds of new generalizations of the poly Dedekind type DC sums. In Sect. 2, for our goal, we show explicit formulas of type 2 unipoly-Euler polynomials and type 2 unipoly-Genocchi polynomials. In Sect. 3, we introduce a unipolyDedekind type DC sum associated with the type 2 unipoly-Euler functions expressed in the type 2 unipoly-Euler polynomials using the modified polyexponential function, and derive the reciprocity relation for these unipoly-Dedekind type DC sums. In Sect. 4, we introduce a unipoly-Dedekind sums type DC associated with the poly-Euler functions expressed in the unipoly-Euler polynomials using the polylogarithm function, and derive the reciprocity relation for those.

The Euler polynomials $E_{n}(x)(n \in \mathbb{N} \cup\{0\})$ are defined by their generating functions as follows:

$$
\frac{2}{e^{t}+1} e^{x t}=\sum_{n=0}^{\infty} E_{n}(x) \frac{t^{n}}{n !} \quad(\text { see }[13,14,19,20])
$$

When $x=0, E_{n}:=E_{n}(0)$ are called Euler numbers. The first few of Euler numbers are $E_{0}=1$, $E_{1}=-\frac{1}{2}, E_{2}=0, E_{3}=\frac{1}{4}, E_{4}=0, \ldots$, and $E_{2 n}=0,(n \in \mathbb{N})$.

The Genocchi polynomials $G_{n}(x),(n \in \mathbb{N} \cup\{0\})$, are defined by their generating functions as follows:

$$
\frac{2 t}{e^{t}+1} e^{x t}=\sum_{n=0}^{\infty} G_{n}(x) \frac{t^{n}}{n !} \quad(\text { see }[9,20,29])
$$


When $x=0, G_{n}:=G_{n}(0)$ are called Genocchi numbers. The first few of Euler numbers are $G_{0}=0, G_{1}=1, G_{2}=-1, G_{3}=0, G_{4}=1, \ldots$, and $G_{2 n+1}=0,(n \in \mathbb{N})$.

We note that

$$
E_{n}=\frac{G_{n+1}}{n+1} \quad(\text { see }[6,13,20]) .
$$

From (2), we note that

$$
E_{n}(x)=\sum_{l=0}^{n}\left(\begin{array}{l}
n \\
l
\end{array}\right) E_{l} x^{n-l}=(E+x)^{n}, \quad(n \geq 0),(\text { see }[13,19,20])
$$

and

$$
G_{n}(x)=\sum_{l=0}^{n}\left(\begin{array}{l}
n \\
l
\end{array}\right) G_{l} x^{n-l}=(G+x)^{n}, \quad(n \geq 0),(\text { see }[12,20]),
$$

with the usual convention about replacing $E^{n}$ and $G^{n}$ with $E_{n}$ and $G_{n}$, respectively.

From $(2)$, for $n \equiv 1(\bmod 2)$, we have

$$
(-1)^{n-1} E_{l}(n)+E_{l}=2 \sum_{i=0}^{n-1}(-1)^{i} i^{l}, \quad(n \in \mathbb{N}),(\text { see }[20])
$$

Let $d$ be an odd positive integer $\geq 3$. Then we have the following well-known relation:

$$
E_{n}(x)=d^{n} \sum_{i=0}^{d-1}(-1)^{i} E_{n}\left(\frac{x+i}{d}\right), \quad(\text { see }[20]),
$$

where $d$ is an odd positive integer $\geq 3$ and $n \geq 0$.

The Euler function is defined by

$$
\bar{E}_{n}(x)=E_{n}(x-[x]), \quad(n \geq 0),(\text { see }[13,20,30]),
$$

where $[x]$ denotes the greatest integer not exceeding $x$.

Kim and Kim considered the modified polyexponential function defined by

$$
\mathrm{Ei}_{k}(x)=\sum_{n=1}^{\infty} \frac{x^{n}}{n^{k}(n-1) !}, \quad(k \in \mathbb{Z}),(\text { see }[10]) .
$$

Note that $\operatorname{Ei}_{1}(x)=e^{x}-1$.

We introduce the type 2 poly-Euler polynomials, which are given by

$$
\frac{\operatorname{Ei}_{k}(\log (1+2 t))}{t\left(e^{t}+1\right)} e^{x t}=\sum_{n=0}^{\infty} E_{k, n}(x) \frac{t^{n}}{n !} \quad(k \in \mathbb{Z}),(\text { see }[19]) .
$$

When $x=0, E_{k, n}=E_{k, n}(0), n \geq 0$, are called type 2 poly-Euler numbers. 
We also introduce the type 2 poly-Genocchi polynomials, which are given by

$$
\frac{\operatorname{Ei}_{k}(\log (1+2 t))}{e^{t}+1} e^{x t}=\sum_{n=0}^{\infty} G_{k, n}(x) \frac{t^{n}}{n !} \quad(k \in \mathbb{Z}),(\text { see }[12]) .
$$

When $x=0, G_{k, n}=G_{k, n}(0), n \geq 0$, are called type 2 poly-Genocchi numbers.

By (12), we easily get $G_{k, 0}=0, G_{k, 1}=1, G_{k, 2}=-2+2^{l-k}, \ldots$. Since $\operatorname{Ei}_{1}(\log (1+2 t))=2 t$, we see that $E_{1, n}(x)=E_{n}(x)$ and $G_{1, n}(x)=G_{n}(x)(n \geq 0)$ are the Euler polynomials and the Genocchi polynomials, respectively.

Kim introduced the Dedekind type DC sums given by

$$
T_{p}(h, m)=2 \sum_{\mu=1}^{m-1}(-1)^{\mu} \frac{\mu}{m} \overline{E_{p}}\left(\frac{h \mu}{m}\right) \quad \text { (see [13]), }
$$

where $\overline{E_{p}}(x)$ is the $p$ th Euler function.

For $p \in \mathbb{N}$ with $p \equiv 1(\bmod 2)$, the reciprocity law of $T_{p}(h, m)$ is given by

$$
\begin{aligned}
& m^{p} T_{p}(h, m)+h^{p} T_{p}(m, h) \\
& \quad=2 \sum_{\mu=0}^{m-1}\left(m h\left(E+\frac{\mu}{m}\right)+m\left(E+h-\left[\frac{h \mu}{m}\right]\right)\right)^{p}+(h E+m E)^{p}+(p+2) E_{p}, \\
& \mu-\left[\frac{h \mu}{m}\right] \equiv 1(\bmod 2), \quad(\text { see }[13,30]),
\end{aligned}
$$

where $h, k$ are relative prime positive integers and

$$
(E h+E m)^{p}=\sum_{l=0}^{p}\left(\begin{array}{l}
p \\
l
\end{array}\right) E_{l} h^{l} E_{p-l} m^{p-l}(\text { see }[13,30]) .
$$

Recently, Ma et al. introduced the poly-Dedekind type DC sums associated with the type 2 poly-Euler functions, which are given by

$$
T_{p}^{(k)}(h, m)=2 \sum_{\mu=1}^{m-1}(-1)^{\mu} \frac{\mu}{m} \bar{E}_{p}^{(k)}(h \mu / m), \quad(h, m, p \in \mathbb{N}),(\text { see [20]) }
$$

where $\bar{E}_{p}(h \mu / m)=E_{p}(\langle h \mu / m\rangle)$.

For $n \in \mathbb{N} \cup\{0\}$, as is well known, the Stirling numbers of the first kind are defined by

$$
(x)_{0}=1,(x)_{n}=\sum_{l=0}^{n} S_{1}(n, l) x^{l} \quad(n \geq 1),
$$

and

$$
\frac{1}{l !}(\log (1+t))^{l}=\sum_{n=l}^{\infty} S_{1}(n, l) \frac{t^{n}}{n !} \quad(n, l \geq 0),(\text { see }[12,15,17]) .
$$


For $n \geq 0$, the Stirling numbers of the second kind are defined by

$$
x^{n}=\sum_{l=0}^{n} S_{2}(n, l)(x)_{l}
$$

and

$$
\frac{1}{k !}\left(e^{t}-1\right)^{k}=\sum_{n=k}^{\infty} S_{2}(n, k) \frac{t^{n}}{n !} \quad(\text { see }[12,15,17]),
$$

where $(x)_{0}=1,(x)_{n}=x(x-1) \ldots(x-n+1),(n \geq 1)$.

\section{Type 2 unipoly-Euler numbers and type 2 unipoly-Genocchi numbers}

Let $\tau$ be any arithmetic function which is real or complex valued and defined on the set of positive integers $\mathbb{N}$. Then Kim and Kim defined the unipoly function attached to polynomials $\tau$ by

$$
u_{k}(x \mid \tau)=\sum_{n=1}^{\infty} \frac{\tau(n) x^{n}}{n^{k}}, \quad(k \in \mathbb{Z}),(\text { see }[10]) .
$$

When $\tau(n)=1, u_{k}(x \mid 1)=\sum_{n=1}^{\infty} \frac{x^{n}}{n^{k}}=L i_{k}(x)$ is the ordinary polylogarithm function.

From (17), we have

$$
\frac{d}{d x} u_{k}(x \mid \tau)=\frac{1}{x} u_{k-1}(x \mid \tau)
$$

Lee et al. introduced the type 2 unipoly-Euler polynomials of index $k$ defined by

$$
\frac{u_{k}(\log (1+2 t) \mid \tau)}{t\left(e^{t}+1\right)} e^{x t}=\sum_{n=0}^{\infty} \mathcal{E}_{k, n, \tau}(x) \frac{t^{n}}{n !} \quad(\text { see [19]) }
$$

When $x=0, \mathcal{E}_{k, n, \tau}:=\mathcal{E}_{k, n, \tau}(0)$ are called type 2 unipoly-Euler numbers.

The type 2 unipoly-Genocchi polynomials of index $k$ are defined by

$$
\frac{u_{k}(\log (1+2 t) \mid \tau)}{e^{t}+1} e^{x t}=\sum_{n=0}^{\infty} \mathbb{G}_{k, n, \tau}(x) \frac{t^{n}}{n !} \quad(\text { see }[12]) .
$$

When $x=0, \mathbb{G}_{k, n, \tau}:=\mathbb{G}_{k, n, \tau}(0)$ are called type 2 unipoly-Genocchi numbers.

For $n \in \mathbb{N} \cup\{0\}$ and $k \in \mathbb{Z}$, let $\tau(n)=\frac{1}{\Gamma(n)}=\frac{1}{(n-1) !}$. Then, from (12) and (19), we have

$$
\begin{aligned}
\sum_{n=0}^{\infty} \mathcal{E}_{k, n, \frac{1}{\Gamma}}(x) \frac{t^{n}}{n !} & =\frac{u_{k}\left(\log (1+2 t) \mid \frac{1}{\Gamma}\right)}{t\left(e^{t}+1\right)} e^{x t} \\
& =\frac{E i_{k}(\log (1+2 t))}{t\left(e^{t}+1\right)} e^{x t}=\sum_{n=0}^{\infty} E_{k, n}(x) \frac{t^{n}}{n !}
\end{aligned}
$$

Thus, from (21) we have

$$
\mathcal{E}_{k, n, \frac{1}{\Gamma}}(x)=E_{k, n}(x)
$$


Similarly, we get

$$
\mathbb{G}_{k, n, \frac{1}{\Gamma}}(x)=G_{k, n}(x) .
$$

Moreover, from (21), we note that

$$
\begin{aligned}
\frac{u_{k}(\log (1+2 t) \mid \tau)}{t\left(e^{t}+1\right)} e^{x t} & =\left(\sum_{l=0}^{\infty} \mathcal{E}_{k, l, \tau} \frac{t^{l}}{l !}\right)\left(\sum_{m=0}^{\infty} \frac{x^{m}}{m !} t^{m}\right) \\
& =\sum_{n=0}^{\infty}\left(\sum_{l=0}^{n}\left(\begin{array}{l}
n \\
l
\end{array}\right) \mathcal{E}_{k, l, \tau} x^{n-l}\right) \frac{t^{n}}{n !}
\end{aligned}
$$

Thus, by (23), we get

$$
\mathcal{E}_{k, n, \tau}(x)=\sum_{l=0}^{n}\left(\begin{array}{l}
n \\
l
\end{array}\right) \mathcal{E}_{k, l, \tau} x^{n-l} \quad(n \geq 0)
$$

In the same way as (23), we have

$$
\mathbb{G}_{k, n, \tau}(x)=\sum_{l=0}^{n}\left(\begin{array}{l}
n \\
l
\end{array}\right) \mathbb{G}_{k, l, \tau} x^{n-l} \quad(n \geq 0) .
$$

Furthermore, by (24) and (25), we have

$$
\frac{d}{d x} \mathcal{E}_{k, n, \tau}(x)=n \mathcal{E}_{k, n-1, \tau}(x) \quad \text { and } \quad \frac{d}{d x} \mathbb{G}_{k, n, \tau}(x)=n \mathbb{G}_{k, n-1, \tau}(x), \quad(n \geq 1) .
$$

Theorem 1 For $n \geq 1$, we have

$$
\mathcal{E}_{k, n, \tau}(x)=\sum_{m=0}^{n} \sum_{l=1}^{m+1}\left(\begin{array}{l}
n \\
m
\end{array}\right) \frac{(l-1) ! 2^{m} \tau(l) S_{1}(m+1, l)}{l^{k-1}(m+1)} E_{n-m}(x), \quad(k \in \mathbb{Z}),
$$

and

$$
\mathbb{G}_{k, n, \tau}(x)=\sum_{m=0}^{n} \sum_{l=1}^{m+1}\left(\begin{array}{l}
n \\
m
\end{array}\right) \frac{(l-1) ! 2^{m} \tau(l) S_{1}(m+1, l)}{l^{k-1}(m+1)} G_{n-m}(x), \quad(k \in \mathbb{Z}) .
$$

Proof From (2), (15), and (19), we have

$$
\begin{aligned}
\sum_{n=0}^{\infty} \mathcal{E}_{k, n, \tau}(x) \frac{t^{n}}{n !} & =\frac{u_{k}(\log (1+2 t) \mid \tau)}{t\left(e^{t}+1\right)} e^{x t}=\frac{1}{2} \frac{2 e^{x t}}{e^{t}+1} \frac{1}{t} \sum_{l=1}^{\infty} \frac{\tau(l)(\log (1+2 t))^{l}}{l^{k}} \\
& =\frac{1}{2} \frac{2 e^{x t}}{e^{t}+1} \frac{1}{t} \sum_{m=1}^{\infty} \sum_{l=1}^{m} \frac{(l-1) ! 2^{m} \tau(l) S_{1}(m, l)}{l^{k-1}} \frac{t^{m}}{m !} \\
& =\sum_{i=0}^{\infty} E_{i}(x) \frac{t^{i}}{i !} \sum_{m=0}^{\infty} \sum_{l=1}^{m+1} \frac{(l-1) ! 2^{m} \tau(l) S_{1}(m+1, l)}{l^{k-1}(m+1)} \frac{t^{m}}{m !} \\
& =\sum_{n=0}^{\infty}\left(\sum_{m=0}^{n} \sum_{l=1}^{m+1}\left(\begin{array}{l}
n \\
m
\end{array}\right) \frac{(l-1) ! 2^{m} \tau(l) S_{1}(m+1, l)}{l^{k-1}(m+1)} E_{n-m}\right) \frac{t^{n}}{n !} .
\end{aligned}
$$


Therefore, from (29) we get identity (27).

By using (3), (15), and (19) in the same way as (29), we also get identity (28).

In particular, we get

$$
\mathcal{E}_{k, n, \tau}=\sum_{m=0}^{n} \sum_{l=1}^{m+1}\left(\begin{array}{l}
n \\
m
\end{array}\right) \frac{(l-1) ! 2^{m} \tau(l) S_{1}(m+1, l)}{l^{k-1}(m+1)} E_{n-m} \quad(k \in \mathbb{Z}),
$$

and the first few of the type 2 unipoly-Euler numbers are $\mathcal{E}_{0, \tau}^{(k)}=\tau(1), \mathcal{E}_{1, \tau}^{(k)}=\frac{1}{2} \tau(1)+$ $\frac{1}{2^{k-1}} \tau(2), \ldots$ In addition,

$$
\mathbb{G}_{k, n, \tau}=\sum_{m=0}^{n} \sum_{l=1}^{m+1}\left(\begin{array}{l}
n \\
m
\end{array}\right) \frac{(l-1) ! 2^{m} \tau(l) S_{1}(m+1, l)}{l^{k-1}(m+1)} G_{n-m} \quad(k \in \mathbb{Z}),
$$

and the first few of the type 2 unipoly-Genocchi numbers are $\mathbb{G}_{k, 0, \tau}=0, \mathbb{G}_{k, 1, \tau}=\tau(1), \ldots$ Since $\mathbb{G}_{k, 0, \tau}=0$, we note that

$$
\begin{aligned}
\sum_{n=0}^{\infty} \mathcal{E}_{k, n, \tau}(x) \frac{t^{n}}{n !} & =\frac{u_{k}(\log (1+2 t) \mid \tau)}{t\left(e^{t}+1\right)} e^{x t} \\
& =\sum_{n=0}^{\infty} \mathbb{G}_{k, n, \tau}(x) \frac{t^{n-1}}{n !}=\sum_{n=1}^{\infty} \mathbb{G}_{k, n, \tau}(x) \frac{t^{n-1}}{n !}=\sum_{n=0}^{\infty} \frac{\mathbb{G}_{k, n+1, \tau}(x)}{n+1} \frac{t^{n}}{n !}
\end{aligned}
$$

Thus, from (30), we have

$$
\mathcal{E}_{k, n, \tau}(x)=\frac{\mathbb{G}_{k, n+1, \tau}(x)}{n+1}
$$

Lemma 2 For $n \geq 1$, we have

$$
\mathbb{G}_{k, n, \tau}(1)+\mathbb{G}_{k, n, \tau}=2^{n} \sum_{m=1}^{n} \frac{m !}{m^{k}} \tau(m) S_{1}(n, m), \quad(k \in \mathbb{Z}),
$$

and

$$
\mathcal{E}_{k, n-1, \tau}(1)+\mathcal{E}_{k, n-1, \tau}=\frac{2^{n}}{n} \sum_{m=1}^{n} \frac{m !}{m^{k}} \tau(m) S_{1}(n, m), \quad(k \in \mathbb{Z}) .
$$

Proof From (20), we have

$$
\begin{aligned}
u_{k}(\log (1+2 t) \mid \tau) & =\left(\sum_{l=0}^{\infty} \mathbb{G}_{k, l, \tau} \frac{t^{l}}{l !}\right)\left(e^{t}+1\right) \\
& =\sum_{n=0}^{\infty}\left(\mathbb{G}_{k, n, \tau}(1)+\mathbb{G}_{k, n, \tau}\right) \frac{t^{n}}{n !}=\sum_{n=1}^{\infty}\left(\mathbb{G}_{k, n, \tau}(1)+\mathbb{G}_{k, n, \tau}\right) \frac{t^{n}}{n !} .
\end{aligned}
$$

On the other hand, from (17), we have

$$
u_{k}(\log (1+2 t) \mid \tau)=\sum_{m=1}^{\infty} \frac{\tau(m)}{m^{k}}(\log (1+2 t))^{m}
$$




$$
\begin{aligned}
& =\sum_{m=1}^{\infty} \frac{\tau(m) m !}{m^{k}} \sum_{n=m}^{\infty} S_{1}(n, m) \frac{2^{n} t^{n}}{n !} \\
& =\sum_{n=1}^{\infty}\left(2^{n} \sum_{m=1}^{n} \frac{m !}{m^{k}} \tau(m) S_{1}(n, m)\right) \frac{t^{n}}{n !}
\end{aligned}
$$

Therefore, by (34) and (35), we obtain identity (32). By using (31), we get identity (33).

Theorem 3 For an odd positive integer $d \geq 3$ and $n \geq 1$, we have

$$
(-1)^{d-1} \mathbb{G}_{k, n, \tau}(d)+\mathbb{G}_{k, n, \tau}=\sum_{a=1}^{n} \sum_{b=1}^{a} \sum_{i=0}^{d-1}\left(\begin{array}{l}
n \\
a
\end{array}\right)(-1)^{i} i^{n-a} \frac{(b-1) ! 2^{a}}{b^{k-1}} \tau(b) S_{1}(a, b)
$$

and

$$
\begin{aligned}
& (-1)^{d-1} \mathcal{E}_{k, n-1, \tau}(d)+\mathcal{E}_{k, n-1, \tau} \\
& =\frac{1}{n}\left(\sum_{a=1}^{n} \sum_{b=1}^{a} \sum_{i=0}^{d-1}\left(\begin{array}{l}
n \\
a
\end{array}\right)(-1)^{i} i^{n-a} \frac{(b-1) ! 2^{a}}{b^{k-1}} \tau(b) S_{1}(a, b)\right) .
\end{aligned}
$$

Proof For an odd positive integer $d \geq 3$, from (15) and (17), we have

$$
\begin{aligned}
& \sum_{i=0}^{d-1}(-1)^{i} e^{i t} u_{k}(\log (1+2 t) \mid \tau) \\
& \quad=\sum_{i=0}^{d-1}(-1)^{i} \sum_{j=0}^{\infty} i^{j} \frac{t^{j}}{j !} \sum_{b=1}^{\infty} \frac{\tau(b)(\log (1+2 t))^{b}}{b^{k}} \\
& \quad=\sum_{j=0}^{\infty}\left(\sum_{i=0}^{d-1}(-1)^{i} i^{j}\right) \frac{t^{j}}{j !} \sum_{a=1}^{\infty}\left(\sum_{b=1}^{a} \frac{b ! 2^{a}}{b^{k}} \tau(b) S_{1}(a, b)\right) \frac{t^{a}}{a !} \\
& \quad=\sum_{n=1}^{\infty}\left(\sum_{a=1}^{n} \sum_{b=1}^{a} \sum_{i=0}^{d-1}\left(\begin{array}{l}
n \\
a
\end{array}\right)(-1)^{i} i^{n-a} \frac{(b-1) ! 2^{a}}{b^{k-1}} \tau(b) S_{1}(a, b)\right) \frac{t^{n}}{n !} .
\end{aligned}
$$

On the other hand,

$$
\begin{aligned}
\sum_{i=0}^{d-1}(-1)^{i} e^{i t} u_{k}(\log (1+2 t) \mid \tau) & =\frac{(-1)^{d-1} e^{d t}+1}{e^{t}+1} u_{k}(\log (1+2 t) \mid \tau) \\
& =\sum_{n=0}^{\infty}\left((-1)^{d-1} \mathbb{G}_{k, n}(d)+\mathbb{G}_{k, n}\right) \frac{t^{n}}{n !}
\end{aligned}
$$

Therefore, by (38) and (39), we obtain identity (36).

Moreover, from (31), we get identity (37).

Theorem 4 For an odd positive integer $d \geq 1$ and $n \geq 0$, we have

$$
\mathbb{G}_{k, n, \tau}(x)=\sum_{l=0}^{n}\left(\begin{array}{l}
n \\
l
\end{array}\right) \sum_{j=1}^{n-l+1} \sum_{i=0}^{d-1}(-1)^{i} d^{l-1} G_{l}\left(\frac{i+x}{d}\right) \frac{(j-1) ! 2^{n-l}}{j^{k-1}(n-l+1)} \tau(j) S_{1}(n-l+1, j) .
$$


Proof From $\sum_{i=0}^{n-1}(-1)^{i} e^{i t}=\frac{(-1)^{n-1} e^{n t}+1}{e^{t}+1},(n \equiv 1(\bmod 2)),(2)$, and (13), we have

$$
\begin{aligned}
& \frac{u_{k}(\log (1+2 t) \mid \tau)}{e^{t}+1} e^{x t} \\
& =\frac{1}{2 d} \sum_{i=0}^{d-1}(-1)^{i} e^{\left(\frac{i+x}{d}\right) d t} \frac{2 d t}{e^{d t}+1} \frac{1}{t} u_{k}(\log (1+2 t) \mid \tau) \\
& =\frac{1}{2 d} \sum_{i=0}^{d-1}(-1)^{i} \sum_{l=0}^{\infty} G_{l}\left(\frac{i+x}{d}\right) \frac{d^{l} t^{l}}{l !} \frac{1}{t} \sum_{j=1}^{\infty} \frac{\tau(j)(\log (1+2 t))^{j}}{j^{k}} \\
& =\sum_{l=0}^{\infty}\left(\sum_{i=0}^{d-1}(-1)^{i} d^{l-1} G_{l}\left(\frac{i+x}{d}\right)\right) \frac{t^{l}}{l !} \sum_{a=1}^{\infty}\left(\sum_{j=1}^{a} \frac{(j-1) ! 2^{a-1}}{j^{k-1}} \tau(j) S_{1}(a, j)\right) \frac{t^{a-1}}{a !} \\
& =\sum_{l=0}^{\infty}\left(\sum_{i=0}^{d-1} d^{l-1}(-1)^{i} G_{l}\left(\frac{i+x}{d}\right)\right) \frac{t^{l}}{l !} \sum_{a=0}^{\infty}\left(\sum_{j=1}^{a+1} \frac{(j-1) ! 2^{a}}{j^{k-1}(a+1)} \tau(j) S_{1}(a+1, j)\right) \frac{t^{a}}{a !} \\
& =\sum_{n=0}^{\infty}\left(\sum_{l=0}^{n}\left(\begin{array}{l}
n \\
l
\end{array}\right)^{n-l+1} \sum_{j=1}^{d-1} \sum_{i=0}^{d}(-1)^{i} d^{l-1} G_{l}\left(\frac{i+x}{d}\right) \frac{(j-1) ! 2^{n-l}}{j^{k-1}(n-l+1)} \tau(j) S_{1}(n-l+1, j)\right.
\end{aligned}
$$

Therefore, by (40), we get the desired result.

Corollary 5 For an odd positive integer $d \geq 1$ and $n \geq 1$, we have

$$
\mathcal{E}_{k, n-1, \tau}(x)=\sum_{l=0}^{n-1} \sum_{j=1}^{n-l} \sum_{i=0}^{d-1}\left(\begin{array}{c}
n-1 \\
l
\end{array}\right)(-1)^{i} d^{l} E_{l}\left(\frac{i+x}{d}\right) \frac{(j-1) ! 2^{n-l-1} \tau(j) S_{1}(n-l, j)}{j^{k-1}(n-l)} .
$$

Proof From (31) and Theorem 4, we have

$$
\begin{aligned}
\mathcal{E}_{k, n-1, \tau}(x) & =\frac{\mathbb{G}_{n, \tau}(x)}{n} \\
& =\frac{1}{n} \sum_{l=1}^{n}\left(\begin{array}{c}
n \\
l
\end{array}\right) \sum_{j=1}^{n-l+1} \sum_{i=0}^{d-1} d^{l-1}(-1)^{i} G_{l}\left(\frac{i+x}{d}\right) \frac{(j-1) ! \tau(j) 2^{n-l}}{j^{k-1}(n-l+1)} S_{1}(n-l+1, j) \\
& =\frac{1}{n} \sum_{l=0}^{n-1} \sum_{j=1}^{n-l} \sum_{i=0}^{d-1}\left(\begin{array}{c}
n \\
l+1
\end{array}\right) d^{l}(-1)^{i} G_{l+1}\left(\frac{i+x}{d}\right) \frac{(j-1) ! 2^{n-l-1}}{j^{k-1}(n-l)} \tau(j) S_{1}(n-l, j) \\
& =\frac{n}{n} \sum_{l=0}^{n-1} \sum_{j=1}^{n-l} \sum_{i=0}^{d-1}\left(\begin{array}{c}
n-1 \\
l
\end{array}\right) d^{l}(-1)^{i} \frac{G_{l+1}\left(\frac{i+x}{d}\right)}{l+1} \frac{(j-1) ! 2^{n-l-1} \tau(j) S_{1}(n-l, j)}{j^{k-1}(n-l)} \\
& =\sum_{l=0}^{n-1} \sum_{j=1}^{n-l} \sum_{i=0}^{d-1}\left(\begin{array}{c}
n-1 \\
l
\end{array}\right)(-1)^{i} d^{l} E_{l}\left(\frac{i+x}{d}\right) \frac{(j-1) ! 2^{n-l-1} \tau(j) S_{1}(n-l, j)}{j^{k-1}(n-l)} .
\end{aligned}
$$

There, from (41), we arrive at the desired result.

Lemma 6 For $l \in \mathbb{N}$ with $l<q$, we have

$$
\left(\begin{array}{l}
q \\
l
\end{array}\right) \mathcal{E}_{k, q-l, \tau}(1)+\left(\begin{array}{c}
q \\
l-1
\end{array}\right) \mathcal{E}_{k, q-l+1, \tau}(1)=\sum_{j=0}^{q}\left(\begin{array}{c}
q-j+1 \\
l
\end{array}\right)\left(\begin{array}{l}
q \\
j
\end{array}\right) \mathcal{E}_{k, j, \tau} .
$$


Proof For $l \in \mathbb{N}$ with $l<q$, we have

$$
\left.\frac{d^{l}}{d x^{l}}\left(x \mathcal{E}_{k, q, \tau}(x)\right)\right|_{x=1}=l !\left(\begin{array}{c}
q \\
l
\end{array}\right) \mathcal{E}_{k, q-l, \tau}(1)+l !\left(\begin{array}{c}
q \\
l-1
\end{array}\right) \mathcal{E}_{k, q-l+1, \tau}(1) .
$$

On the other hand, by (24), we get

$$
\begin{aligned}
\left.\frac{d^{l}}{d x^{l}}\left(x \mathcal{E}_{k, q, \tau}(x)\right)\right|_{x=1} & =\left.\sum_{j=0}^{q}\left(\begin{array}{c}
q \\
j
\end{array}\right) \mathcal{E}_{k, j, \tau}\left(\left(\frac{d^{l}}{d x^{l}}\right)^{l} x^{q-j+1}\right)\right|_{x=1} \\
& =l ! \sum_{j=0}^{q}\left(\begin{array}{c}
q-j+1 \\
l
\end{array}\right)\left(\begin{array}{l}
q \\
j
\end{array}\right) \mathcal{E}_{k, j, \tau} .
\end{aligned}
$$

Therefore, by (42) and (43), we obtain what we want.

Lemma 7 For $q \in \mathbb{N}$, we have

$$
\sum_{j=0}^{q}\left(\begin{array}{l}
q \\
j
\end{array}\right) \frac{\mathcal{E}_{k, j, \tau}}{q-j+2}=\frac{1}{q+1} \mathcal{E}_{k, q, \tau}(1)-\frac{1}{(q+1)(q+2)} \mathcal{E}_{k, q+2}(1)+\frac{\mathcal{E}_{k, q+2, \tau}}{(q+1)(q+2)} .
$$

Proof By using (26), we observe that

$$
\begin{aligned}
\int_{0}^{1} x \mathcal{E}_{k, q, \tau}(x) d x & =\frac{1}{q+1} \mathcal{E}_{k, q+1}(1)-\frac{1}{q+1} \int_{0}^{1} \mathcal{E}_{k, q+1, \tau}(x) d x \\
& =\frac{1}{q+1} \mathcal{E}_{k, q+1, \tau}(1)-\frac{1}{(q+1)(q+2)}\left(\mathcal{E}_{k, q+2, \tau}(1)-\mathcal{E}_{k, q+2, \tau}\right) .
\end{aligned}
$$

On the other hand, by using (24), we have

$$
\int_{0}^{1} x \mathcal{E}_{k, q, \tau}(x) d x=\sum_{j=0}^{q}\left(\begin{array}{l}
q \\
j
\end{array}\right) \mathcal{E}_{k, j, \tau} \int_{0}^{1} x^{q-j+1} d x=\sum_{j=0}^{q}\left(\begin{array}{l}
q \\
j
\end{array}\right) \frac{\mathcal{E}_{k, j, \tau}}{q-j+2} .
$$

Therefore, by (44) and (45), we get what we want.

\section{Unipoly-Dedekind type DC sums associated withthe type 2 unipoly-Euler functions of index $k$}

In this section, as a generalization of the poly-Dedekind type DC sums, we consider the unipoly-Dedekind type DC sums associated with the type 2 unipoly-Euler functions of index $k$ and derive several noble identities and the reciprocity relation for these.

Naturally, we consider the unipoly-Dedekind type DC sums associated with the type 2 unipoly-Euler functions of index $k$ as follows:

$$
Z_{k, q, \tau}(h, m)=2 \sum_{\mu=1}^{m-1}(-1)^{\mu}(\mu / m) \overline{\mathcal{E}}_{k, q, \tau}(h \mu / m), \quad(h, m, q \in \mathbb{N}, k \in \mathbb{Z}),
$$

where $h, m, q \in \mathbb{N}$ with $q \equiv 1(\bmod 2)$ and $\overline{\mathcal{E}}_{k, q, \tau}(x)=\mathcal{E}_{k, q, \tau}(x-[x])$ are the type 2 unipolyEuler functions of index $k([x]$ is the largest integer less than $x)$. 
For $n \in \mathbb{N} \cup\{0\}$ and $k \in \mathbb{Z}$, when $\tau(n)=\frac{1}{\Gamma(n)}=\frac{1}{(n-1) !}$, we note that $Z_{k, q, \frac{1}{\Gamma}}(h, m)=T_{q}^{(k)}(h, m)$. In addition, we note that

$$
Z_{1, q, \frac{1}{\Gamma}}(h, m)=2 \sum_{\mu=1}^{m-1}(-1)^{\mu}(\mu / m) \overline{\mathcal{E}}_{1, q, \frac{1}{\Gamma}}(h \mu / m)=T_{q}(h, m) .
$$

Theorem 8 Let $m$ be an odd positive integer $\geq 1$ and $q \in \mathbb{N}$. Then we have

$$
m^{q} Z_{k, q, \tau}(1, m)=\sum_{j=0}^{q} \sum_{i=0}^{q-j}\left(\begin{array}{c}
q \\
j
\end{array}\right)\left(\begin{array}{c}
q-j+1 \\
i
\end{array}\right) \mathcal{E}_{k, j, \tau} m^{q-i} E_{i}+2 \sum_{j=0}^{q}\left(\begin{array}{l}
q \\
j
\end{array}\right) \mathcal{E}_{k, j, \tau} m^{j-1} E_{q-j+1}
$$

Proof From (5), (7), and (46), we have

$$
\begin{aligned}
Z_{k, q, \tau}(1, m) \\
=2 \sum_{\mu=0}^{m-1}(-1)^{\mu}\left(\frac{\mu}{m}\right) \overline{\mathcal{E}}_{k, q, \tau}\left(\frac{\mu}{m}\right)=2 \sum_{\mu=0}^{m-1}(-1)^{\mu}\left(\frac{\mu}{m}\right) \mathcal{E}_{k, q, \tau}\left(\frac{\mu}{m}\right) \\
=2 \sum_{\mu=0}^{m-1}(-1)^{\mu}\left(\frac{\mu}{m}\right) \sum_{j=0}^{q}\left(\begin{array}{l}
q \\
j
\end{array}\right)\left(\frac{\mu}{m}\right)^{q-j} \mathcal{E}_{k, j, \tau} \\
=\sum_{j=0}^{q}\left(\begin{array}{l}
q \\
j
\end{array}\right) \mathcal{E}_{k, j, \tau} m^{-(q-j+1)}\left(2 \sum_{\mu=0}^{m-1} \mu^{q-j+1}(-1)^{\mu}\right) \\
=\sum_{j=0}^{q}\left(\begin{array}{l}
q \\
j
\end{array}\right) \mathcal{E}_{k, j, \tau} m^{-q+j-1}\left((-1)^{m-1} E_{q-j+1}(m)+E_{q-j+1}\right) \\
=\sum_{j=0}^{q}\left(\begin{array}{l}
q \\
j
\end{array}\right) \mathcal{E}_{k, j, \tau} m^{-q+j-1}\left(\sum_{i=0}^{q-j+1}\left(\begin{array}{c}
q+1-j \\
i
\end{array}\right) m^{q-j+1-i} E_{i}+E_{q-j+1}\right) \\
=\sum_{j=0}^{q}\left(\begin{array}{l}
q \\
j
\end{array}\right) \mathcal{E}_{k, j, \tau} m^{-q+j-1} \sum_{i=0}^{q-j}\left(\begin{array}{c}
q-j+1 \\
i
\end{array}\right) m^{q-j+1-i} E_{i}+2 \sum_{j=0}^{q}\left(\begin{array}{l}
q \\
j
\end{array}\right) \mathcal{E}_{k, j, \tau} m^{-q+j-1} E_{q-j+1} \\
=\sum_{j=0}^{q} \sum_{i=0}^{q-j}\left(\begin{array}{c}
q \\
j
\end{array}\right)\left(\begin{array}{c}
q-j+1 \\
i
\end{array}\right) \mathcal{E}_{k, j, \tau} m^{-i} E_{i}+2 \sum_{j=0}^{q}\left(\begin{array}{l}
q \\
j
\end{array}\right) \mathcal{E}_{k, j, \tau} m^{-q+j-1} E_{q-j+1} .
\end{aligned}
$$

By multiplying both sides of (47) by $m^{q}$, we arrive at the desired result.

Theorem 9 Let $m, q$ be odd positive integers $m \geq 1$ and $q \geq 3$, respectively. Then we have

$$
\begin{aligned}
& m^{q} Z_{k, q, \tau}(1, m) \\
& =\sum_{i=1}^{q-2}\left(\begin{array}{l}
q \\
i
\end{array}\right) \mathcal{E}_{k, q-i, \tau}(1) m^{q-i} E_{i}+\sum_{i=1}^{q-2}\left(\begin{array}{c}
q \\
i-1
\end{array}\right)\left(\mathcal{E}_{k, q-i+1, \tau}(1)-\mathcal{E}_{k, q-i+1, \tau}\right) m^{q-i} E_{i} \\
& \quad+2 \sum_{j=0}^{q}\left(\begin{array}{l}
q \\
j
\end{array}\right) \mathcal{E}_{k, j, \tau} m^{j-1} E_{q-j+1}+(q+1) \tau(1) E_{q}+m^{q} \mathcal{E}_{k, q, \tau}(1) .
\end{aligned}
$$


Proof For an odd integer $q \geq 3$, we observe that $E_{q-1}=0$. Moreover, $E_{0}=1$ and $\mathcal{E}_{k, 0, \tau}=\tau(1)$. From (24), we observe that

$$
\begin{aligned}
\sum_{j=0}^{q} & \sum_{i=0}^{q-j}\left(\begin{array}{c}
q \\
j
\end{array}\right)\left(\begin{array}{c}
q-j+1 \\
i
\end{array}\right) \mathcal{E}_{k, j, \tau} m^{q-i} E_{i} \\
= & \sum_{i=0}^{q} \sum_{j=0}^{q-i}\left(\begin{array}{l}
q \\
j
\end{array}\right)\left(\begin{array}{c}
q-j+1 \\
i
\end{array}\right) \mathcal{E}_{k, j, \tau} m^{q-i} E_{i} \\
= & \sum_{j=0}^{q}\left(\begin{array}{l}
q \\
j
\end{array}\right) \mathcal{E}_{k, j, \tau} m^{q}+\sum_{i=1}^{q-2} \sum_{j=0}^{q-i}\left(\begin{array}{l}
q \\
j
\end{array}\right)\left(\begin{array}{c}
q-j+1 \\
i
\end{array}\right) \mathcal{E}_{k, j, \tau} m^{q-i} E_{i} \\
& +\sum_{j=0}^{1}\left(\begin{array}{l}
q \\
j
\end{array}\right)\left(\begin{array}{c}
q-j+1 \\
q-1
\end{array}\right) \mathcal{E}_{k, j, \tau} m E_{q-1}+\left(\begin{array}{c}
q+1 \\
q
\end{array}\right) \tau(1) E_{q} \\
= & \sum_{i=1}^{q-2} \sum_{j=0}^{q-i}\left(\begin{array}{l}
q \\
j
\end{array}\right)\left(\begin{array}{c}
q-j+1 \\
i
\end{array}\right) \mathcal{E}_{k, j, \tau} m^{q-i} E_{i}+(q+1) \tau(1) E_{q}+m^{q} \mathcal{E}_{k, q, \tau}(1) .
\end{aligned}
$$

In addition, by using Lemma 6, we have

$$
\begin{aligned}
\sum_{j=0}^{q-i}\left(\begin{array}{c}
q \\
j
\end{array}\right)\left(\begin{array}{c}
q-j+1 \\
i
\end{array}\right) \mathcal{E}_{k, j, \tau} & =\sum_{j=0}^{q}\left(\begin{array}{l}
q \\
j
\end{array}\right)\left(\begin{array}{c}
q-j+1 \\
i
\end{array}\right) \mathcal{E}_{k, j, \tau}-\left(\begin{array}{c}
q \\
i-1
\end{array}\right) \mathcal{E}_{k, q-i+1, \tau} \\
& =\left(\begin{array}{c}
q \\
i
\end{array}\right) \mathcal{E}_{k, q-i, \tau}(1)+\left(\begin{array}{c}
q \\
i-1
\end{array}\right) \mathcal{E}_{k, q-i+1, \tau}(1)-\left(\begin{array}{c}
q \\
i-1
\end{array}\right) \mathcal{E}_{k, q-i+1, \tau} \\
& =\left(\begin{array}{c}
q \\
i
\end{array}\right) \mathcal{E}_{k, q-i, \tau}(1)+\left(\begin{array}{c}
q \\
i-1
\end{array}\right)\left(\mathcal{E}_{k, q-i+1, \tau}(1)-\mathcal{E}_{k, q-i+1, \tau}\right) .
\end{aligned}
$$

Therefore, from Theorem 8, (48), and (49), we get

$$
\begin{aligned}
& m^{q} Z_{k, q, \tau}(1, m) \\
& =\sum_{i=1}^{q-2}\left(\begin{array}{c}
q \\
i
\end{array}\right) \mathcal{E}_{k, q-i, \tau}(1) m^{q-i} E_{i}+\sum_{i=1}^{q-2}\left(\begin{array}{c}
q \\
i-1
\end{array}\right)\left(\mathcal{E}_{k, q-i+1, \tau}(1)-\mathcal{E}_{k, q-i+1, \tau}\right) m^{q-i} E_{i} \\
& \quad+2 \sum_{j=0}^{q}\left(\begin{array}{c}
q \\
j
\end{array}\right) \mathcal{E}_{k, j, \tau} m^{j-1} E_{q-j+1}+(q+1) \tau(1) E_{q}+m^{q} \mathcal{E}_{k, q, \tau}(1) .
\end{aligned}
$$

To prove the next theorem, we employ the symbolic notations as $E_{n}(x)=(E+x)^{n}$, $\mathcal{E}_{k, n, \tau}(x)=\left(\mathcal{E}_{k, \tau}+x\right)^{n},(n \geq 0)$, with the usual convention about replacing $E^{n}$ and $\left(\mathcal{E}_{k, \tau}\right)^{n}$ with $E_{n}$ and $\mathcal{E}_{k, n, \tau}$, respectively.

Theorem 10 Let $h, m$ be relatively prime positive integers and $m, q$ be odd positive integers $m \geq 1$ and $q \geq 3$, respectively. Then we have

$$
m^{q} \sum_{\alpha=0}^{m-1} \sum_{j=0}^{q}(-1)^{\alpha}\left(\begin{array}{l}
q \\
j
\end{array}\right) \mathcal{E}_{k, j, \tau}\left(\frac{\alpha}{m}\right) h^{j} E_{q-j}\left(h-\left[\frac{h \alpha}{m}\right]\right)=\sum_{j=0}^{q}\left(\begin{array}{l}
q \\
j
\end{array}\right)(m h)^{q-j} E_{j} \mathcal{E}_{k, q-j, \tau}(1) .
$$


Proof As the index $\alpha$ ranges through the values $\alpha=0,1,2, \ldots, m-1$, the product $h \alpha$ ranges over a complete residue system modulo $m$ such that $h, m$ are relatively prime positive integers, and we may replace $<\frac{h \alpha}{m}>=\frac{h \alpha}{m}-\left[\frac{h \alpha}{m}\right]$ with $<\frac{h \alpha}{m}>$ without alternating the sum over $\alpha$.

Therefore, from (8), we observe that

$$
\begin{aligned}
m^{q} & \sum_{\alpha=0}^{m-1}(-1)^{\alpha} \sum_{j=0}^{q}\left(\begin{array}{l}
q \\
j
\end{array}\right) \mathcal{E}_{k, j, \tau}\left(\frac{\alpha}{m}\right) h^{j} E_{q-j}\left(h-\left[\frac{h \alpha}{m}\right]\right) \\
& =m^{q} \sum_{\alpha=0}^{m-1}(-1)^{a}\left(h\left(\mathcal{E}_{k, \tau}+\frac{\alpha}{m}\right)+\left(E+h-\left[\frac{h \alpha}{m}\right]\right)\right)^{q} \\
& =m^{q} \sum_{\alpha=0}^{m-1}(-1)^{\alpha}\left(h \mathcal{E}_{k, \tau}+E+h+\frac{1}{2}-\frac{1}{2}+\frac{h \alpha}{m}-\left[\frac{h \alpha}{m}\right]\right)^{q} \\
& =m^{q} \sum_{\alpha=0}^{m-1}(-1)^{\alpha}\left(h \mathcal{E}_{k, \tau}+E+h+\frac{1}{2}+\bar{E}_{1}\left(\frac{\alpha}{m}\right)\right)^{q} \\
& =m^{q} \sum_{\alpha=0}^{m-1}(-1)^{\alpha}\left(h\left(\mathcal{E}_{k, \tau} 1\right)+E+\frac{\alpha}{m}\right)^{q} \\
& =m^{q} \sum_{\alpha=0}^{m-1}(-1)^{\alpha} \sum_{j=0}^{q}\left(\begin{array}{l}
q \\
j
\end{array}\right)\left(E+\frac{\alpha}{m}\right)^{j} h^{q-j}\left(\mathcal{E}_{k, \tau}+1\right)^{q-j} \\
& =\sum_{j=0}^{q}\left(\begin{array}{l}
q \\
j
\end{array}\right)(m h)^{q-j} E_{j} \mathcal{E}_{k, q-j, \tau}(1) . \\
& =m^{q} \sum_{\alpha=0}^{m-1}(-1)^{\alpha} \sum_{j=0}^{q}\left(\begin{array}{l}
q \\
j
\end{array}\right) E_{j}\left(\frac{\alpha}{m}\right)^{q-j} \mathcal{E}_{k, q-j, \tau}(1) \\
& =\sum_{j=0}^{q}\left(\begin{array}{l}
q \\
j
\end{array}\right) m^{q-j}\left(m^{j} \sum_{\alpha=0}^{m-1}(-1)^{\alpha} E_{s}\left(\frac{\alpha}{m}\right) h^{q-j} \mathcal{E}_{k, q-j, \tau}(1)\right. \\
&
\end{aligned}
$$

Therefore, from (50), we obtain what we want.

Theorem 11 Let $m, q$ be odd positive integers $m \geq 1$ and $q \geq 3$, respectively. Then we have

$$
\begin{aligned}
& m^{q} Z_{k, q, \tau}(h, m)+h^{q} Z_{k, q, \tau}(m, h) \\
& =\sum_{\alpha=0}^{m-1} \sum_{\beta=0}^{h-1} \sum_{l=0}^{q} \sum_{j=1}^{q+1-l}\left(\begin{array}{l}
q \\
l
\end{array}\right)(-1)^{\alpha+\beta-1} \frac{(j-1) ! \tau(j) 2^{q-l+1} S_{1}(q+1-l, j)}{j^{k-1}(q+1-l)} \\
& \quad \times\left((\alpha h) m^{q-l}+(\beta m) h^{q-l}\right) \overline{E_{l}}\left(\frac{\alpha}{m}+\frac{\beta}{h}\right) .
\end{aligned}
$$

Proof From Corollary 5, we note that

$$
\overline{\mathcal{E}}_{k, q, \tau}(x)=\sum_{l=0}^{q} \sum_{j=1}^{q} \sum_{i=0}^{d-1}\left(\begin{array}{l}
q \\
l
\end{array}\right)(-1)^{i} d^{l} \bar{E}_{l}\left(\frac{i+x}{d}\right) \frac{(j-1) ! 2^{q-l} \tau(j) S_{1}(q+1-l, j)}{j^{k-1}(q+1-l)},
$$

where $d \in \mathbb{N}$ with $d \equiv 1(\bmod 2), k \in \mathbb{Z}$, and $n \geq 0$. 
From (46) and (51), we get

$$
\begin{aligned}
& m^{q} Z_{k, q, \tau}(h, m)+h^{q} Z_{k, q, \tau}(m, h) \\
& =2 m^{q} \sum_{\alpha=0}^{m-1}(-1)^{\alpha} \frac{\alpha}{m} \overline{\mathcal{E}}_{k, q, \tau}\left(\frac{h \alpha}{m}\right)+2 h^{q} \sum_{\beta=0}^{h-1}(-1)^{\beta} \frac{\beta}{h} \overline{\mathcal{E}}_{k, q, \tau}\left(\frac{m \beta}{h}\right) \\
& =2 m^{q} \sum_{\alpha=0}^{m-1}(-1)^{\alpha} \frac{\alpha}{m} \sum_{l=0}^{q} \sum_{j=1}^{q+1-l} \sum_{\beta=0}^{h-1}\left(\begin{array}{l}
q \\
l
\end{array}\right)(-1)^{\beta} h^{l} \overline{E_{l}}\left(\frac{\beta}{h}+\frac{\alpha}{m}\right) \\
& \times \frac{(j-1) ! 2^{q-l} \tau(j) S_{1}(q+1-l, j)}{j^{k-1}(q+1-l)} \\
& +2 h^{q} \sum_{\beta=0}^{h-1}(-1)^{\beta} \frac{\beta}{h} \sum_{l=0}^{q} \sum_{j=1}^{q+1-l} \sum_{\alpha=0}^{m-1}\left(\begin{array}{l}
q \\
l
\end{array}\right)(-1)^{\alpha} m^{l} \overline{E_{l}}\left(\frac{\alpha}{m}+\frac{\beta}{h}\right) \\
& \times \frac{(j-1) ! 2^{q-l} \tau(j) S_{1}(q+1-l, j)}{j^{k-1}(q+1-l)} \\
& =2 \sum_{\alpha=0}^{m-1} \sum_{l=0}^{q} \sum_{j=1}^{q+1-l} \sum_{\beta=0}^{h-1}\left(\begin{array}{l}
q \\
l
\end{array}\right)(-1)^{\alpha+\beta} \frac{\alpha}{m} m^{q-l}(m h)^{l} \overline{E_{l}}\left(\frac{\beta}{h}+\frac{\alpha}{m}\right) \\
& \times \frac{(j-1) ! 2^{q-l} \tau(j) S_{1}(q+1-l, j)}{j^{k-1}(q+1-l)} \\
& +2 \sum_{\beta=0}^{h-1} \sum_{l=0}^{q} \sum_{j=1}^{q+1-l} \sum_{\alpha=0}^{m-1}\left(\begin{array}{l}
q \\
l
\end{array}\right)(-1)^{\alpha+\beta} \frac{\beta}{h} h^{q-l}(m h)^{l} \overline{E_{l}}\left(\frac{\alpha}{m}+\frac{\beta}{h}\right) \\
& \times \frac{(j-1) ! 2^{q-l} \tau(j) S_{1}(q+1-l, j)}{j^{k-1}(q+1-l)} \\
& =\sum_{\alpha=0}^{m-1} \sum_{l=0}^{q} \sum_{j=1}^{q+1-l} \sum_{\beta=0}^{h-1}\left(\begin{array}{l}
q \\
l
\end{array}\right)(-1)^{\alpha+\beta}(\alpha h)(m h)^{-1} m^{q-l}(m h)^{l} \overline{E_{l}}\left(\frac{\beta}{h}+\frac{\alpha}{m}\right) \\
& \times \frac{(j-1) ! 2^{q-l+1} \tau(j) S_{1}(q+1-l, j)}{j^{k-1}(q+1-l)} \\
& +\sum_{\beta=0}^{h-1} \sum_{l=0}^{q} \sum_{j=1}^{q+1-l} \sum_{\alpha=0}^{m-1}\left(\begin{array}{l}
q \\
l
\end{array}\right)(-1)^{\alpha+\beta}(\beta m)(m h)^{-1} h^{q-l}(m h)^{l} \overline{E_{l}}\left(\frac{\alpha}{m}+\frac{\beta}{h}\right) \\
& \times \frac{(j-1) ! 2^{q-l+1} \tau(j) S_{1}(q+1-l, j)}{j^{k-1}(q+1-l)} \\
& =\sum_{\alpha=0}^{m-1} \sum_{l=0}^{q} \sum_{j=1}^{q+1-l} \sum_{\beta=0}^{h-1}\left(\begin{array}{l}
q \\
l
\end{array}\right)(-1)^{\alpha+\beta} \frac{(j-1) ! \tau(j) 2^{q-l+1} S_{1}(q+1-l, j)}{j^{k-1}(q+1-l)} \\
& \times\left((\alpha h) m^{q-l}+(\beta m) h^{q-l}\right) \overline{E_{l}}\left(\frac{\alpha}{m}+\frac{\beta}{h}\right) .
\end{aligned}
$$


Corollary 12 Let $m, q$, be odd positive integers $m \geq 1$ and $q \geq 3$, respectively. Then we get

$$
\begin{aligned}
m^{q} Z_{1, q, \frac{1}{\Gamma}}(h, m)+h^{q} Z_{1, q, \frac{1}{\Gamma}}(m, h) & =m^{q} T_{q}(h, m)+h^{q} T_{q}(m, h) \\
& =2 \sum_{\alpha=0}^{m-1} \sum_{\beta=0}^{h-1}(m h)^{q-1}(-1)^{\alpha+\beta}(\alpha h+m \beta) \bar{E}_{q}\left(\frac{\beta}{h}+\frac{\alpha}{m}\right),
\end{aligned}
$$

where $\Gamma(n)=(n-1)$ !.

\section{Unipoly-Dedekind type DC sums associated withunipoly-Euler functions of index $k$}

In this section, as another generalization of the poly Dedekind type DC sums, we consider unipoly-Dedekind type DC sums associated with the unipoly-Euler functions of index $k$ and derive the reciprocity relation for these. For the purposes of this section, we first introduce two new polynomials, the poly-Euler polynomials and poly-Genocchi polynomials, using the polylogarithm function of arbitrary index $k$.

It is well known that the polylogarithm function of index $k$ is defined by

$$
L i_{k}(x)=\sum_{n=1}^{\infty} \frac{x^{n}}{n^{k}}, \quad(k \in \mathbb{Z}),(\text { see }[8,10,12]) .
$$

Note that $L i_{1}(x)=-\log (1-x)$.

We consider the poly-Euler polynomials given by

$$
\frac{L i_{k}\left(1-e^{-2 t}\right)}{t\left(e^{t}+1\right)} e^{x t}=\sum_{n=0}^{\infty} E_{k, n}^{*}(x) \frac{t^{n}}{n !}, \quad(k \in \mathbb{Z}) .
$$

When $x=0, E_{k, n}^{*}=E_{k, n}^{*}(0), n \geq 0$, are called poly-Euler numbers.

We also introduce the poly-Genocchi polynomials, which are given by

$$
\frac{L i_{k}\left(1-e^{-2 t}\right)}{e^{t}+1} e^{x t}=\sum_{n=0}^{\infty} G_{k, n}^{*}(x) \frac{t^{n}}{n !}, \quad(k \in \mathbb{Z}) .
$$

When $x=0, G_{k, n}^{*}=G_{k, n}^{*}(0), n \geq 0$, are called poly-Genocchi numbers.

Since $L i_{1}\left(1-e^{-2 t}\right)=2 t$, we see that $E_{n}^{*(1)}(x)=E_{n}(x)$ and $G_{n}^{*(1)}(x)=G_{n}(x)(n \geq 0)$ are the Euler polynomials and the Genocchi polynomials, respectively.

In addition, we define the unipoly-Euler polynomials of arbitrary index $k$ defined by

$$
\frac{\left.u_{k}\left(1-e^{-2 t}\right) \mid \tau\right)}{t\left(e^{t}+1\right)} e^{x t}=\sum_{n=0}^{\infty} E_{k, n, \tau}^{*}(x) \frac{t^{n}}{n !} .
$$

When $x=0, E_{k, n, \tau}^{*}=E_{k, n, \tau}^{*}(0)$ are called unipoly-Euler numbers.

When $\tau(n)=1$ for all $n, E_{k, n, 1}^{*}(x)=E_{k, n}^{*}(x)$ is the poly-Euler polynomials.

The unipoly-Genocchi polynomials of arbitrary index $k$ are defined by

$$
\frac{\left.u_{k}\left(1-e^{-2 t}\right) \mid \tau\right)}{e^{t}+1} e^{x t}=\sum_{n=0}^{\infty} G_{k, n, \tau}^{*}(x) \frac{t^{n}}{n !} .
$$

When $x=0, \mathbb{G}_{k, n, \tau}^{*}=\mathbb{G}_{k, n, \tau}^{*}(0)$ are called unipoly-Genocchi numbers. 
When $\tau(n)=1$ for all $n, G_{k, n, 1}^{*}(x)=G_{k, n}^{*}(x)$ is the poly-Genocchi polynomials.

Now, we consider a new type of unipoly-Dedekind type DC sums associated with the unipoly-Euler function of index $k$ as follows:

$$
Y_{k, q, \tau}(h, m)=2 \sum_{\mu=1}^{m-1}(-1)^{\mu}(\mu / m) \bar{E}_{k, q, \tau}^{*}(h \mu / m), \quad(h, m, q \in \mathbb{N}, k \in \mathbb{Z}),
$$

where $h, m, q \in \mathbb{N}$ with $q \equiv 1(\bmod 2)$ and $\bar{E}_{k, q, \tau}^{*}(x)=E_{k, q, \tau}^{*}(x-[x])$ are the unipoly-Euler functions of index $k([x]$ is the largest integer less than $x$ ).

For $n \in \mathbb{N} \cup\{0\}$ and $k \in \mathbb{Z}$, let $\tau(n)=1$. From $\bar{E}_{k, q, 1}^{*}=\bar{E}_{k, q}^{*}$ and (13), we note that $Y_{k, q, 1}(h, m)=T_{k, q}(h, m)$.

$$
Y_{1, q, 1}(h, m)=2 \sum_{\mu=1}^{m-1}(-1)^{\mu}(\mu / m) \bar{E}_{1, q, 1}(h \mu / m)=T_{q}(h, m) .
$$

We note that

$$
\frac{\left.u_{k}\left(1-e^{-2 t}\right) \mid \tau\right)}{t\left(e^{t}+1\right)} e^{x t}=\left(\sum_{l=0}^{\infty} E_{k, l, \tau}^{*} \frac{t^{l}}{l !}\right)\left(\sum_{m=0}^{\infty} \frac{x^{m}}{m !} t^{m}\right)=\sum_{n=0}^{\infty}\left(\sum_{l=0}^{n}\left(\begin{array}{l}
n \\
l
\end{array}\right) E_{k, l, \tau}^{*} x^{n-l}\right) \frac{t^{n}}{n !} .
$$

Thus, by (58), we get

$$
E_{k, n, \tau}^{*}(x)=\sum_{l=0}^{n}\left(\begin{array}{l}
n \\
l
\end{array}\right) E_{k, l, \tau}^{*} x^{n-l} \quad(n \geq 0) .
$$

In the same way as (59), we have

$$
G_{k, n, \tau}^{*}(x)=\sum_{l=0}^{n}\left(\begin{array}{l}
n \\
l
\end{array}\right) G_{k, l, \tau}^{*} x^{n-l} \quad(n \geq 0)
$$

Furthermore, by (59) and (60), we have

$$
\frac{d}{d x} E_{k, n, \tau}^{*}(x)=n E_{k, n-1, \tau}^{*}(x) \quad \text { and } \quad \frac{d}{d x} G_{k, n, \tau}^{*}(x)=n G_{k, n-1, \tau}^{*}(x), \quad(n \geq 1) .
$$

Theorem 13 For $n \geq 1$, we have

$$
E_{k, n, \tau}^{*}(x)=\sum_{m=0}^{n} \sum_{l=1}^{m+1}\left(\begin{array}{l}
n \\
m
\end{array}\right) \frac{(l-1) !(-1)^{l+m+1} 2^{m} \tau(l) S_{2}(m+1, l)}{l^{k-1}(m+1)} E_{n-m}(x), \quad(k \in \mathbb{Z})
$$

and

$$
G_{k, n, \tau}^{*}(x)=\sum_{m=0}^{n} \sum_{l=1}^{m+1}\left(\begin{array}{c}
n \\
m
\end{array}\right) \frac{(l-1) !(-1)^{l+m+1} 2^{m} \tau(l) S_{2}(m+1, l)}{l^{k-1}(m+1)} G_{n-m}(x), \quad(k \in \mathbb{Z}) .
$$

Proof From (2), (16), and (55), we have

$$
\sum_{n=0}^{\infty} E_{k, n, \tau}^{*}(x) \frac{t^{n}}{n !}=\frac{\left.u_{k}\left(1-e^{-2 t}\right) \mid \tau\right)}{t\left(e^{t}+1\right)} e^{x t}=\frac{1}{2} \frac{2 e^{x t}}{e^{t}+1} \frac{1}{t} \sum_{l=1}^{\infty} \frac{\tau(l)\left(1-e^{-2 t}\right)^{l}}{l^{k}}
$$




$$
\begin{aligned}
& =\frac{1}{2} \frac{2 e^{x t}}{e^{t}+1} \frac{1}{t} \sum_{m=1}^{\infty} \sum_{l=1}^{m} \frac{(l-1) !(-1)^{l}(-2)^{m} \tau(l) S_{2}(m, l)}{l^{k-1}} \frac{t^{m}}{m !} \\
& =\sum_{i=0}^{\infty} E_{i}(x) \frac{t^{i}}{i !} \sum_{m=0}^{\infty} \sum_{l=1}^{m+1} \frac{(l-1) !(-1)^{l+m+1} 2^{m} \tau(l) S_{2}(m+1, l)}{l^{k-1}(m+1)} \frac{t^{m}}{m !} \\
& =\sum_{n=0}^{\infty}\left(\sum_{m=0}^{n} \sum_{l=1}^{m+1}\left(\begin{array}{l}
n \\
m
\end{array}\right) \frac{(l-1) !(-1)^{l+m+1} 2^{m} \tau(l) S_{2}(m+1, l)}{l^{k-1}(m+1)} E_{n-m}(x)\right) \frac{t^{n}}{n !} .
\end{aligned}
$$

Therefore, from (64), we get identity (62).

By using (3), (16), and (56), in the same way as (64), we also get identity (63).

In (62), when $x=1$, we get

$$
E_{k, n, \tau}^{*}=\sum_{m=0}^{n} \sum_{l=1}^{m+1}\left(\begin{array}{l}
n \\
m
\end{array}\right) \frac{(l-1) !(-1)^{l+m+1} 2^{m} \tau(l) S_{2}(m+1, l)}{l^{k-1}(m+1)} E_{n-m} \quad(k \in \mathbb{Z}),
$$

and the first few of the unipoly-Euler numbers are $E_{k, 0, \tau}^{*}=\tau(1), E_{k, 1, \tau}^{*}=-\tau(1)+\frac{1}{2^{k-1}} \tau(2), \ldots$ In (63), when $x=1$, we get

$$
G_{k, n, \tau}^{*}=\sum_{m=0}^{n} \sum_{l=1}^{m+1}\left(\begin{array}{l}
n \\
m
\end{array}\right) \frac{(l-1) !(-1)^{l+m+1} 2^{m} \tau(l) S_{2}(m+1, l)}{l^{k-1}(m+1)} G_{n-m} \quad(k \in \mathbb{Z}),
$$

and the first few of the unipoly-Genocchi numbers are $G_{k, 0, \tau}^{*}=0, G_{k, 1, \tau}^{*}=\tau(1), \ldots$

Since $G_{k, 0, \tau}^{*}=0$, we note that

$$
E_{k, n, \tau}^{*}(x)=\frac{G_{k, n+1, \tau}^{*}(x)}{n+1} .
$$

Lemma 14 For $n \geq 1$, we have

$$
G_{k, n, \tau}^{*}(1)+G_{k, n, \tau}^{*}=2^{n} \sum_{m=1}^{n} \frac{(-1)^{n+m} m !}{m^{k}} \tau(m) S_{2}(n, m), \quad(k \in \mathbb{Z})
$$

and

$$
E_{k, n-1, \tau}^{*}(1)+E_{k, n-1, \tau}^{*}=\frac{2^{n}}{n} \sum_{m=1}^{n} \frac{(-1)^{n+m} m !}{m^{k}} \tau(m) S_{2}(n, m), \quad(k \in \mathbb{Z}) .
$$

Proof From (5) and (56), we have

$$
\begin{aligned}
u_{k}\left(1-e^{-2 t} \mid \tau\right) & =\left(\sum_{l=0}^{\infty} G_{k, l, \tau}^{*} \frac{t^{l}}{l !}\right)\left(e^{t}+1\right) \\
& =\sum_{n=0}^{\infty}\left(G_{k, n, \tau}^{*}(1)+G_{k, n, \tau}^{*}\right) \frac{t^{n}}{n !} \\
& =\sum_{n=1}^{\infty}\left(G_{k, n, \tau}^{*}(1)+G_{k, n, \tau}^{*}\right) \frac{t^{n}}{n !} .
\end{aligned}
$$


On the other hand, from (17), we have

$$
\begin{aligned}
u_{k}\left(1-e^{-2 t} \mid \tau\right) & =\sum_{m=1}^{\infty} \frac{\tau(m)}{m^{k}}(-1)^{m}\left(e^{-2 t}-1\right)^{m} \\
& =\sum_{m=1}^{\infty} \frac{\tau(m) m !}{m^{k}}(-1)^{m} \sum_{n=m}^{\infty} S_{2}(n, m) \frac{(-2)^{n} t^{n}}{n !} \\
& =\sum_{n=1}^{\infty}\left(2^{n} \sum_{m=1}^{n} \frac{(-1)^{n+m} m !}{m^{k}} \tau(m) S_{2}(n, m)\right) \frac{t^{n}}{n !} .
\end{aligned}
$$

Therefore, by (70) and (71), we obtain identity (68). By using (67), we get identity (69)

Theorem 15 For an odd positive integer $d \geq 3$ and $n \geq 1$, we have

$$
\begin{aligned}
& (-1)^{d-1} G_{k, n, \tau}(d)+G_{k, n, \tau}^{*} \\
& =\sum_{a=1}^{n} \sum_{b=0}^{a} \sum_{i=0}^{d-1}\left(\begin{array}{l}
n \\
a
\end{array}\right)(-1)^{i} i^{n-a} \frac{(b-1) !(-1)^{a+b} 2^{a}}{b^{k-1}} \tau(b) S_{2}(a, b)
\end{aligned}
$$

and

$$
\begin{aligned}
& (-1)^{d-1} E_{k, n-1, \tau}^{*}(d)+E_{k, n-1, \tau}^{*} \\
& \quad=\frac{1}{n}\left(\sum_{a=1}^{n} \sum_{b=0}^{a} \sum_{i=0}^{d-1}\left(\begin{array}{l}
n \\
a
\end{array}\right)(-1)^{i} i^{n-a} \frac{(b-1) !(-1)^{a+b} 2^{a}}{b^{k-1}} \tau(b) S_{2}(a, b)\right) .
\end{aligned}
$$

Proof For an odd positive integer $d \geq 3$, from (15) and (17), we have

$$
\begin{aligned}
& \sum_{i=0}^{d-1}(-1)^{i} e^{i t} u_{k}\left(1-e^{-2 t} \mid \tau\right) \\
& \quad=\sum_{i=0}^{d-1}(-1)^{i} \sum_{j=0}^{\infty} i^{j} \frac{t^{j}}{j !} \sum_{b=1}^{\infty} \frac{\tau(b)(-1)^{b}\left(e^{-2 t}-1\right)^{b}}{b^{k}} \\
& \quad=\sum_{j=0}^{\infty}\left(\sum_{i=0}^{d-1}(-1)^{i} i^{j}\right) \frac{t^{j}}{j !} \sum_{b=1}^{\infty} \frac{\tau(b)(-1)^{b} b !}{b^{k}} \sum_{a=b}^{\infty} S_{2}(a, b) \frac{(-2 t)^{a}}{a !} \\
& \quad=\sum_{j=0}^{\infty}\left(\sum_{i=0}^{d-1}(-1)^{i} i^{j}\right) \frac{t^{j}}{j !} \sum_{a=1}^{\infty}\left(\sum_{b=1}^{a} \frac{(b-1) !(-1)^{b}(-2)^{a}}{b^{k-1}} \tau(b) S_{2}(a, b)\right) \frac{t^{a}}{a !} \\
& \quad=\sum_{n=1}^{\infty}\left(\sum_{a=1}^{n} \sum_{b=0}^{a} \sum_{i=0}^{d-1}\left(\begin{array}{l}
n \\
a
\end{array}\right)(-1)^{i} i^{n-a} \frac{(b-1) !(-1)^{a+b} 2^{a}}{b^{k-1}} \tau(b) S_{2}(a, b)\right) \frac{t^{n}}{n !}
\end{aligned}
$$

On the other hand, from $\sum_{i=0}^{n-1}(-1)^{i} e^{i t}=\frac{(-1)^{n-1} e^{n t}+1}{e^{t}+1},(n \equiv 1(\bmod 2))$, we have

$$
\sum_{i=0}^{d-1}(-1)^{i} e^{i t} u_{k}\left(1-e^{-2 t} \mid \tau\right)=\frac{(-1)^{d-1} e^{d t}+1}{e^{t}+1} u_{k}\left(1-e^{-2 t} \mid \tau\right)
$$




$$
=\sum_{n=0}^{\infty}\left((-1)^{d-1} G_{k, n}^{*}(d)+G_{k, n}^{*}\right) \frac{t^{n}}{n !}
$$

Therefore, by (74) and (75), we obtain identity (72).

Moreover, from (67), we get identity (73).

Theorem 16 For an odd positive integer $d \geq 1$ and $n \geq 0$, we have

$$
\begin{aligned}
G_{k, n, \tau}^{*}(x)= & \sum_{l=0}^{n}\left(\begin{array}{l}
n \\
l
\end{array}\right) \sum_{j=1}^{n-l+1} \sum_{i=0}^{d-1}(-1)^{i} d^{l-1} \\
& \times G_{l}\left(\frac{i+x}{d}\right) \frac{(j-1) !(-1)^{j+n-l+1} 2^{n-l}}{j^{k-1}(n-l+1)} \tau(j) S_{2}(n-l+1, j) .
\end{aligned}
$$

Proof From (2) and (14), for an odd positive integer $d \geq 1$, we have

$$
\begin{aligned}
& \frac{u_{k}\left(1-e^{-2 t} \mid \tau\right)}{e^{t}+1} e^{x t} \\
& =\frac{1}{2 d} \sum_{i=0}^{d-1}(-1)^{i} e^{\left(\frac{i+x}{d}\right) d t} \frac{2 d t}{e^{d t}+1} \frac{1}{t} u_{k}\left(1-e^{-2 t} \mid \tau\right) \\
& =\frac{1}{2 d} \sum_{i=0}^{d-1}(-1)^{i} \sum_{l=0}^{\infty} G_{l}\left(\frac{i+x}{d}\right) \frac{d^{l} t^{l}}{l !} \frac{1}{t} \sum_{j=1}^{\infty} \frac{\left.\tau(j)(-1)^{j}\left(e^{-2 t}-1\right)\right)^{j}}{j^{k}} \\
& =\frac{1}{2 d} \sum_{i=0}^{d-1}(-1)^{i} \sum_{l=0}^{\infty} G_{l}\left(\frac{i+x}{d}\right) \frac{d^{l} t^{l}}{l !} \frac{1}{t} \sum_{j=1}^{\infty} \frac{\tau(j)(-1)^{j} j !}{j^{k}} \sum_{a=j}^{\infty} S_{2}(a, j) \frac{(-2 t)^{a}}{a !} \\
& =\sum_{l=0}^{\infty}\left(\sum_{i=0}^{d-1}(-1)^{i} d^{l-1} G_{l}\left(\frac{i+x}{d}\right)\right) \frac{t^{l}}{l !} \sum_{a=1}^{\infty}\left(\sum_{j=1}^{a} \frac{(j-1) !(-1)^{j+a} 2^{a-1}}{j^{k-1}} \tau(j) S_{2}(a, j)\right) \frac{t^{a-1}}{a !} \\
& =\sum_{l=0}^{\infty}\left(\sum_{i=0}^{d-1} d^{l-1}(-1)^{i} G_{l}\left(\frac{i+x}{d}\right)\right) \frac{t^{l}}{l !} \sum_{a=0}^{\infty}\left(\sum_{j=1}^{a+1} \frac{(j-1) !(-1)^{j+a+1} 2^{a}}{j^{k-1}(a+1)} \tau(j) S_{2}(a+1, j)\right) \frac{t^{a}}{a !} \\
& =\sum_{n=0}^{\infty}\left(\sum_{l=0}^{n}\left(\begin{array}{l}
n \\
l
\end{array}\right) \sum_{j=1}^{n-l+1} \sum_{i=0}^{d-1}(-1)^{i} d^{l-1}\right. \\
& \left.\times G_{l}\left(\frac{i+x}{d}\right) \frac{(j-1) !(-1)^{j+n-l+1} 2^{n-l}}{j^{k-1}(n-l+1)} \tau(j) S_{2}(n-l+1, j)\right) \frac{t^{n}}{n !} .
\end{aligned}
$$

Therefore, by (76), we get the desired result.

Corollary 17 For an odd positive integer $d \geq 1$ and $n \geq 1$, we have

$$
E_{k, n-1, \tau}^{*}(x)=\sum_{l=0}^{n-1} \sum_{j=1}^{n-l} \sum_{i=0}^{d-1}\left(\begin{array}{c}
n-1 \\
l
\end{array}\right)(-1)^{i} d^{l} E_{l}\left(\frac{i+x}{d}\right) \frac{(j-1) !(-1)^{n+j-l} 2^{n-l-1} \tau(j) S_{2}(n-l, j)}{j^{k-1}(n-l)} .
$$


Proof From (67) and Theorem 16, we have

$$
\begin{aligned}
& E_{k, n-1, \tau}^{*}(x) \\
& =\frac{G_{k, n, \tau}^{*}(x)}{n} \\
& =\frac{1}{n} \sum_{l=1}^{n}\left(\begin{array}{c}
n \\
l
\end{array}\right) \sum_{j=1}^{n-l+1} \sum_{i=0}^{d-1} d^{l-1}(-1)^{i} G_{l}\left(\frac{i+x}{d}\right) \frac{(j-1) ! \tau(j)(-1)^{j+n-l+1} 2^{n-l}}{j^{k-1}(n-l+1)} S_{2}(n-l+1, j) \\
& =\frac{1}{n} \sum_{l=0}^{n-1} \sum_{j=1}^{n-l} \sum_{i=0}^{d-1}\left(\begin{array}{c}
n \\
l+1
\end{array}\right) d^{l}(-1)^{i} G_{l+1}\left(\frac{i+x}{d}\right) \frac{(j-1) !(-1)^{j+n-l} 2^{n-l-1}}{j^{k-1}(n-l)} \tau(j) S_{2}(n-l, j) \\
& =\frac{n}{n} \sum_{l=0}^{n-1} \sum_{j=1}^{n-l} \sum_{i=0}^{d-1}\left(\begin{array}{c}
n-1 \\
l
\end{array}\right) d^{l}(-1)^{i} \frac{G_{l+1}\left(\frac{i+x}{d}\right)}{l+1} \frac{(j-1) !(-1)^{j+n-l} 2^{n-l-1} \tau(j) S_{2}(n-l, j)}{j^{k-1}(n-l)} \\
& =\sum_{l=0}^{n-1} \sum_{j=1}^{n-l} \sum_{i=0}^{d-1}\left(\begin{array}{c}
n-1 \\
l
\end{array}\right)(-1)^{i} d^{l} E_{l}\left(\frac{i+x}{d}\right) \frac{(j-1) !(-1)^{n+j-l} 2^{n-l-1} \tau(j) S_{2}(n-l, j)}{j^{k-1}(n-l)} .
\end{aligned}
$$

There, from (77), we arrive at the desired result.

We can obtain the following lemmas in the same way as Lemma 6 and Lemma 7, respectively, in Sect. 2.

Lemma 18 For $l \in \mathbb{N}$ with $l<q$, we have

$$
\left(\begin{array}{l}
q \\
l
\end{array}\right) E_{k, q-l, \tau}^{*}(1)+\left(\begin{array}{c}
q \\
l-1
\end{array}\right) E_{k, q-l+1, \tau}^{*}(1)=\sum_{j=0}^{q}\left(\begin{array}{c}
q-j+1 \\
l
\end{array}\right)\left(\begin{array}{l}
q \\
j
\end{array}\right) E_{k, j, \tau}^{*} .
$$

Lemma 19 For $q \in \mathbb{N}$, we have

$$
\sum_{j=0}^{q}\left(\begin{array}{l}
q \\
j
\end{array}\right) \frac{E_{k, j, \tau}^{*}}{q-j+2}=\frac{1}{q+1} E_{k, q, \tau}^{*}(1)-\frac{1}{(q+1)(q+2)} E_{k, q+2, \tau}^{*}(1)+\frac{E_{k, q+2, \tau}^{*}}{(q+1)(q+2)}
$$

In addition, we can obtain the following theorems in the same way as Theorem 9 and Theorem 10, respectively, in Sect. 3.

Theorem 20 Let $m, q$ be odd positive integers $m \geq 1$ and $q \geq 3$, respectively. Then we have

$$
\begin{aligned}
& m^{q} Y_{k, q, \tau}(1, m) \\
& =\sum_{i=1}^{q-2}\left(\begin{array}{l}
q \\
i
\end{array}\right) E_{k, q-i, \tau}^{*}(1) m^{q-i} E_{i}+\sum_{i=1}^{q-2}\left(\begin{array}{c}
q \\
i-1
\end{array}\right)\left(E_{k, q-i+1, \tau}^{*}(1)-E_{k, q-i+1, \tau}^{*}\right) m^{q-i} E_{i} \\
& \quad+2 \sum_{j=0}^{q}\left(\begin{array}{l}
q \\
j
\end{array}\right) E_{k, j, \tau}^{*} m^{j-1} E_{q-j+1}+(q+1) \tau(1) E_{q}+m^{q} E_{k, q, \tau}^{*}(1) .
\end{aligned}
$$


Theorem 21 Let $h, m$ be relatively prime positive integers and $m, q$ be odd positive integers $m \geq 1$ and $q \geq 3$, respectively. Then we have

$$
m^{q} \sum_{\alpha=0}^{m-1} \sum_{j=0}^{q}(-1)^{\alpha}\left(\begin{array}{l}
q \\
j
\end{array}\right) E_{k, j, \tau}^{*}\left(\frac{\alpha}{m}\right) h^{j} E_{q-j}\left(h-\left[\frac{h \alpha}{m}\right]\right)=\sum_{j=0}^{q}\left(\begin{array}{l}
q \\
j
\end{array}\right)(m h)^{q-j} E_{j} E_{k, q-j, \tau}^{*}(1) .
$$

Now, we obtain the following reciprocity theorem for the unipoly-Dedekind type DC sums associated with the unipoly-Euler function with index $k$.

Theorem 22 Let $m, q$ be odd positive integers $m \geq 1$ and $q \geq 3$, respectively. Then we have

$$
\begin{aligned}
& m^{q} Y_{k, q, \tau}(h, m)+h^{q} Y_{k, q, \tau}(m, h) \\
& =\sum_{\alpha=0}^{m-1} \sum_{\beta=0}^{h-1} \sum_{l=0}^{q} \sum_{j=1}^{q+1-l}\left(\begin{array}{l}
q \\
l
\end{array}\right)(-1)^{\alpha+\beta+q+1+j-l} \frac{(j-1) ! \tau(j) 2^{q-l+1} S_{2}(q+1-l, j)}{j^{k-1}(q+1-l)} \\
& \quad \times\left((\alpha h) m^{q-l}+(\beta m) h^{q-l}\right) \overline{E_{l}}\left(\frac{\alpha}{m}+\frac{\beta}{h}\right) .
\end{aligned}
$$

Proof From Corollary 17, we note that

$$
\begin{aligned}
\bar{E}_{k, q, \tau}^{*}(x)= & \sum_{l=0}^{q} \sum_{j=1}^{q} \sum_{i=0}^{d-1}\left(\begin{array}{l}
q \\
l
\end{array}\right)(-1)^{i} d^{l} \bar{E}_{l}\left(\frac{i+x}{d}\right) \\
& \times \frac{(j-1) !(-1)^{q+1+j-l} 2^{q-l} \tau(j) S_{2}(q+1-l, j)}{j^{k-1}(q+1-l)},
\end{aligned}
$$

where $d \in \mathbb{N}$ with $d \equiv 1(\bmod 2), k \in \mathbb{Z}$, and $n \geq 0$.

From (78), in the same way as (53),

$$
\begin{aligned}
m^{q} Y_{k, q, \tau}(h, m)+h^{q} Y_{k, q, \tau}(m, h) \\
=2 m^{q} \sum_{\alpha=0}^{m-1}(-1)^{\alpha} \frac{\alpha}{m} \bar{E}_{k, q, \tau}\left(\frac{h \alpha}{m}\right)+2 h^{q} \sum_{\beta=0}^{h-1}(-1)^{\beta} \frac{\beta}{h} \bar{E}_{k, q, \tau}\left(\frac{m \beta}{h}\right) \\
=2 m^{q} \sum_{\alpha=0}^{m-1}(-1)^{\alpha} \frac{\alpha}{m} \sum_{l=0}^{q} \sum_{j=1}^{q+1-l} \sum_{\beta=0}^{h-1}\left(\begin{array}{l}
q \\
l
\end{array}\right)(-1)^{\beta} h^{l} \bar{E}_{l}\left(\frac{\beta}{h}+\frac{\alpha}{m}\right) \\
\quad \times \frac{(j-1) !(-1)^{q+1+j-l} 2^{q-l} \tau(j) S_{2}(q+1-l, j)}{j^{k-1}(q+1-l)} \\
+2 h^{q} \sum_{\beta=0}^{h-1}(-1)^{\beta} \frac{\beta}{h} \sum_{l=0}^{q} \sum_{j=1}^{q+1-l} \sum_{\alpha=0}^{m-1}\left(\begin{array}{l}
q \\
l
\end{array}\right)(-1)^{\alpha} m^{l} \overline{E_{l}}\left(\frac{\alpha}{m}+\frac{\beta}{h}\right) \\
\quad \times \frac{(j-1) !(-1)^{q+1+j-l} 2^{q-l} \tau(j) S_{2}(q+1-l, j)}{j^{k-1}(q+1-l)} \\
=2 \sum_{\alpha=0}^{m-1} \sum_{l=0}^{q} \sum_{j=1}^{q+1-l} \sum_{\beta=0}^{h-1}\left(\begin{array}{l}
q \\
l
\end{array}\right)(-1)^{\alpha+\beta} \frac{\alpha}{m} m^{q-l}(m h)^{l} \overline{E_{l}}\left(\frac{\beta}{h}+\frac{\alpha}{m}\right) \\
\quad \times \frac{(j-1) !(-1)^{q+1+j-l} 2^{q-l} \tau(j) S_{2}(q+1-l, j)}{j^{k-1}(q+1-l)}
\end{aligned}
$$




$$
\begin{aligned}
& +2 \sum_{\beta=0}^{h-1} \sum_{l=0}^{q} \sum_{j=1}^{q+1-l} \sum_{\alpha=0}^{m-1}\left(\begin{array}{l}
q \\
l
\end{array}\right)(-1)^{\alpha+\beta} \frac{\beta}{h} h^{q-l}(m h)^{l} \overline{E_{l}}\left(\frac{\alpha}{m}+\frac{\beta}{h}\right) \\
& \times \frac{(j-1) !(-1)^{q+1+j-l} 2^{q-l} \tau(j) S_{2}(q+1-l, j)}{j^{k-1}(q+1-l)} \\
& =\sum_{\alpha=0}^{m-1} \sum_{l=0}^{q} \sum_{j=1}^{q+1-l} \sum_{\beta=0}^{h-1}\left(\begin{array}{l}
q \\
l
\end{array}\right)(-1)^{\alpha+\beta+q+1+j-l} \frac{(j-1) ! \tau(j) 2^{q-l+1} S_{2}(q+1-l, j)}{j^{k-1}(q+1-l)} \\
& \times\left((\alpha h) m^{q-l}+(\beta m) h^{q-l}\right) \overline{E_{l}}\left(\frac{\alpha}{m}+\frac{\beta}{h}\right) .
\end{aligned}
$$

Therefore, from (79), we obtain the reciprocity relation for the unipoly-Dedekind type DC sums.

Corollary 23 Let $m, q$, be odd positive integers $m \geq 1$ and $q \geq 3$, respectively. Then we get

$$
\begin{aligned}
m^{q} Y_{1, q, 1}(h, m)+h^{q} Y_{1, q, 1}(m, h) & =m^{q} T_{q}(h, m)+h^{q} T_{q}(m, h) \\
& =2 \sum_{\alpha=0}^{m-1} \sum_{\beta=0}^{h-1}(m h)^{q-1}(-1)^{\alpha+\beta}(\alpha h+m \beta) \bar{E}_{q}\left(\frac{\beta}{h}+\frac{\alpha}{m}\right),
\end{aligned}
$$

where $\tau(n)=1$ for all $n$.

\section{Conclusion}

In this paper, as further generalizations of the poly-Dedekind type DC sums, we introduced two kinds of unipoly-Dedekind type DC sums. In Sect. 3, the type 2 unipolyDedekind type DC sums associated with the type 2 unipoly-Euler functions of index $k$ were introduced, and some interesting identities and the reciprocity relation were shown. In Sect. 4, the unipoly-Dedekind type DC sums associated with the unipoly-Euler functions of index $k$ were introduced, and some interesting identities and the reciprocity relation were shown. We would like to further study another Dedekind type DC sums.

\section{Acknowledgements}

The authors would like to thank the referees for the detailed and valuable comments that helped improve the original manuscript in its present form. Also, the authors thank Jangjeon Institute for Mathematical Science for the support of this research.

\section{Funding}

This work was supported by the Basic Science Research Program, the National Research Foundation of Korea (NRF-2021R1F1A1050151).

\section{Availability of data and materials}

Not applicable.

Ethics approval and consent to participate

The authors declare that there is no ethical problem in the production of this paper.

Competing interests

The authors declare that there is no competing interests regarding the publication of this article. 


\section{Author details}

'Department of Mathematics Education, Daegu Catholic University, Gyeongsan 38430, Republic of Korea. ${ }^{2}$ School of Electronic and Electric Engineering, Daegu University, Gyeongsan 38453, Republic of Korea.

\section{Publisher's Note}

Springer Nature remains neutral with regard to jurisdictional claims in published maps and institutional affiliations.

Received: 14 March 2021 Accepted: 24 June 2021 Published online: 17 July 2021

\section{References}

1. Apostol, T.M.: Generalized Dedekind sums and transformation formulae of certain Lambert series. Duke Math. J. 17, 147-157 (1950)

2. Apostol, T.M.: Theorems on generalized Dedekind sums. Pac. J. Math. 2, 1-9 (1952)

3. Bayad, A.: On values of Jacobi forms and elliptic Dedekind sums. Proc. Jangjeon Math. Soc. 12(2), 119-139 (2009)

4. Bayad, A.: Jacobi forms in two variables: multiple elliptic Dedekind sums, the Kummer-Von Syaudt Clausen congruences for elliptic Bernoulli functions and values of Hecke L-functions. Montes Taurus. J. Pure Appl. Math. 1(2), 58-129 (2019)

5. Beck, M., Diaz, R., Robins, S.: The Frobenius problem, rational polytopes, and Fourier Dedekind sums. J. Number Theory 96, 1-21 (2002)

6. Can, M., Cenkci, M., Kurt, V.I., Simsek, Y.: Twisted Dedekind type sums associated with Barnes' type multiple Frobenius-Euler I-functions. Adv. Stud. Contemp. Math. (Kyungshang) 18(2), 135-160 (2009)

7. Cangul, I.N., Ozden, H., Simsek, Y.: A new approach to q-Genocchi numbers and their interpolation functions. Nonlinear Anal. 71, 793-799 (2009)

8. Carlitz, L.: Some theorems on generalized Dedekind-Rademacher sums. Pac. J. Math. 75(2), 347-358 (1978)

9. Dedekind, R.: Erlauterungen zu Riemann's Fragmenten uber die Grenzfalle der elliptischen Funktionen, vol. 1, pp. 159-173. Gesammelte Mathematische Werke, Braunschweig (1930)

10. Kim, D.S., Kim, T.: A note on polyexponential and unipoly functions. Russ. J. Math. Phys. 26(1), 40-49 (2019)

11. Kim, H.K.: A note on unipoly Dedekind sums. https://doi.org/10.13140/R.G.2.2.21982.25926

12. Kim, H.K., Jang, L.-C.: A note on degenerate poly-Genocchi numbers and polynomials. Adv. Differ. Equ. 2020, 392 (2020)

13. Kim, T.: Note on Dedekind type DC sums. Adv. Stud. Contemp. Math. (Kyungshang) 18(2), 249-260 (2009)

14. Kim, T.: Note on q-Dedekind-type sums related to q-Euler polynomials. Glasg. Math. J. 54(1), 121-125 (2012)

15. Kim, T., Kim, D.S.: Degenerate polyexponential functions and degenerate Bell polynomials. J. Math. Anal. Appl. 487(2), $124017(2020)$

16. Kim, T., Kim, D.S., Jang, L.-C., Lee, H., Kim, H.: Generalized degenerate Bernoulli numbers and polynomials arising from Gauss hypergeometric function. Adv. Differ. Equ. 2021, 175 (2021)

17. Kim, T., Kim, D.S., Kwon, J., Lee, H.: Degenerate polyexponential functions and type 2 degenerate poly-Bernoulli numbers and polynomials. Adv. Differ. Equ. 2020, 168 (2020)

18. Kim, T., Kim, D.S., Lee, H., Jang, L.-C.: Identities on poly-Dedekind sums. Adv. Differ. Equ. 2020, 563 (2020)

19. Lee, D.S., Kim, H.K., Jang, L.-C.: Type 2 degenerate poly-Euler polynomials. Symmetry 1011, 12 (2020)

20. Ma, Y., Kim, D.S., Lee, H., Kim, H., Kim, T.: Reciprocity of poly-Dedekind-type DC sums involving poly-Euler fuctions. Adv. Differ. Equ. 2021, 30 (2021)

21. Ma, Y., Kim, D.S., Lee, H., Kim, T.: Poly-Dedekind sums with poly-Bernoulli functions. J. Inequal. Appl. 2020, 248 (2020)

22. Meyer, C.: Uber einige Anwendungen Dedekindscher Summen. J. Reine Angew. Math. 198, 143-203 (1957)

23. Milovanovic, G., Simsek, Y.: Dedekind and Hardy Type Sums and Trigonometric Sums Induced by Quadrature Formulas. Trigonometric Sums and Their Applications, 183-228 (2020) (eBook ISBN 978-3-030-37904-9)

24. Mordell, L.J.: Lattice points in a tetrahedron and generalized Dedekind sums. J. Indian Math. Soc. 15, 41-46 (1951)

25. Rademacher, H.: Zur Theorie der Modulfunktionen. J. Reine Angew. Math. 167, 312-336 (1932)

26. Rademacher, H.: Generalization of the reciprocity formular for Dedekind sums. Duke Math. J. 21, $391-397$ (1954)

27. Rademacher, H., Whiteman, A.: Theorems on Dedekind sum. Am. J. Math. 63, 377-407 (1941)

28. Simsek, Y.: Remarks on reciprocity laws of the Dedekind and Hardy sums. Adv. Stud. Contemp. Math. (Kyungshang) $12(2), 237-246(2006)$

29. Simsek, Y.: q-Hardy-Berndt type sums associated with q-Genocchi type zeta and q-I-functions. Nonlinear Anal. 71 377-395 (2009)

30. Simsek, Y.: Special functions related to Dedekind-type DC-sums and their applications. Russ. J. Math. Phys. 17(4), 495-508 (2010) 